the basic physico-chemical parameters can be given and a real approach is made to the derivation of corrections for the pressure applicable to homogenization temperatures. There is also a useful paper by V. A. Kalyushnyi on the construction of homogenization curves and their use as records of changes of phase ratio resulting from heating, where the theoretical basis is considered.

The techniques are likely to be of growing importance in work on mineral paragenesis and the origin of ore deposits. This book is by far the most comprehensive and important text on their development and use which has so far appeared in any language.

K. C. Dunham

\section{MECHANICS OF FLUIDS}

\section{Mechanics of Liquids and Gases}

By L. G. Loitsyanskii. (International Series of Monographs in Aeronautics and Astronautics. Division II: Aerodynamics.) Pp. xii +802 . (London and New York: Pergamon Press, Ltd., 1966.) 160s. net.

ON the whole, the approach in this book is conventional -beginning with kinematics, going on to the discussion of the flow of inviscid fluids and ending with accounts of viscous fluids and of turbulent flows. There is also a most interesting introduction, which is unusual in a book of this kind.

There is a great deal of mathematics in the book, and it is possible for the reader to get lost among the many equations strung along his way, though if he persists he will be brought firmly back to the appropriate physical problem in the end. The difficulties are increased partly because of a certain inconsistency in the methods used (there is, for example, a section on tensors which are then only intermittently used in the text), and partly because the theory is developed first in the abstract for the general case, and it is not until later that the simplifications are made which enable the reader to see its application to particular problems. For example, Loitsyanskii develops the three-dimensional equations of motion before discuss. ing the problem of motion in one dimension.

Thero is very little discussion of the physical nature of fluids and of the microscopic causes of the internal forces. Further, although at least half the book is concerned with viseous and turbulent flows, it is possible to read the first half without realizing why it is that viscous forces and turbulence can often be ignored: the matter is dealt with in three or four lines.

Thero are several misprints in the book, but these should cause no difficulty to the careful reader who checks his formulae. A more serious problem for the English reader using tho book for reference is that it sometimes happens that formulae are given different names from those used in Britain: an example of this is the Mangler transformation which, in one form, is referred to here as Stepanov's transformation. This merely means that the reador should check his references elsewhere.

It is obviously for reference that the book will bo primarily used, and it will be of particular value to teachers who are preparing a course on some of the subject matter in the book. There is a wealth of mathematical detail, some exceptionally clear descriptions (for example, that of a vortex line in Chapter 1) and some excellent diagrams.

Ruth H. ROgERS

\section{HIGH RESOLUTION NMR}

\section{Théories Moléculaires de la Résonance Magnétique Nucléaire}

Applications a la Chimie Structurale. Par G. Mavel. Pp. $\mathrm{x}+325$. (Paris: Dunod, 1966.) 78 francs.

Professor MAvel has restricted his subjoct matter to thooretical descriptions of NMR phenomena and the various techniques available, and an outline of the quantum mechanical calculations of chemical shifts and coupling constants, parameters that can be obtained from NMR spectra. He has discussed very fow applications, but to compensate for this has included a very complete list of references. Ho has not attempted to describe the various spectrometers available or the procedures adopted to obtain spectra.

The subject matter can be divided into three sections: the derivation of the spectral parameters (chemical shifts and coupling constants) from spectra; the theoretical estimation of these quantities; and the effects of chemical exchange. The first of these will probably be of greatest interest to readers; it describes most of the methods now available and provides extensive tables. Double and multi-resonance experiments can be performod, or multiple transitions observed in attempts to reduce the number of conceivable spectral assignments. The theories of these effects are very well reviewed when they are pertinent to spectral interpretation, but where the emphasis is on the study of relaxation phenomena discussion is brief. The derivation of every possible assignment and the determ. ination of optimum parameters for each is work well suited to a computer, and a number of programs are now available. These are listed and their functions described, but there is no discussion of their operation. The second section describing the derivation of chemical shifts and coupling constants is a very good literature survey, but discussion is necessarily brief. The third section satisfactorily describes the effects of chemical exchange on spectrum appearance, but the determination of exchange rates by pulse methods is only referred to.

In summary, this book is oriented towards workers using conventional high resolution NMR techniques on hydrogen and fluorine nuclei, and outlines the dovelopments that are relevant to their work, while reference is made to developments in fringe topics. Experienced workers will presumably use the book as a handbook and reference source, and newcomers to the field will find the literature survey extremely valuable. W. Derbyshire

\section{PRECIPITATORS}

\section{An Introduction to Electrostatic Precipitation in Theory and Practice}

By H. F. Rose and A. J. Wood. Second edition, revised and enlarged. Pp. 212. (London: Constable and Co., Ltd., 1966.) 30s. net.

THIs is the second edition of a well known monograph. first published in 1956. It deals with the removal of solid particles or liquid droplets from a gas in which they are suspended, by giving the particles an electric charge, then removing them from the gas by means of an electric field. As the authors state, the phenomena taking place are ill understood and design methods are largely empirical.

After a description of the various types of equipment an expression for the theoretical efficiency of a precipitator is derived. The authors have used the usual exponential form of efficiency equation first proposed by Deutsch. In this they are clearly in a dilomma, since they produce photographic evidence that the assumption on which the formula is based is not valid. Perhaps they might have mentioned the work of Little ${ }^{1}$, whose results of tests on a large scale precipitator suggest that the Deutsch formula is not obeyed, and of Williams and Jackson ${ }^{2}$, who attempted to produce an alternative theory.

Some problems arising in the design and operation of precipitators are then discussed, including the effects of the electrical resistivity of the dust, the use of conditioning agents and the avoidance of back ionization and corona suppression. A chapter is devoted to the mechanical design and construction of precipitators with particular roforenco to rapping mechanisms and electrode design. 